\title{
A Study on the Effect of Learning Strategy using a Highlighter Pen on Gaze Movement
}

\author{
Hiroki Nishimura \\ Graduate School of Science and Technology \\ Kyoto Institute of Technology \\ Kyoto, Japan
}

\author{
Noriaki Kuwahara \\ Graduate School of Science and Technology \\ Kyoto Institute of Technology \\ Kyoto, Japan
}

\begin{abstract}
In this study, we propose a learning strategy using a highlighter pen to improve the learning efficiency of learners. This method makes the important information stand out by colouring text. It is known that highlighting important points of sentence problems with a highlighter pen improves the speed of answers and correct answer rates, especially in school subjects, such as Japanese and mathematics. In this study, we focused on the gaze movement and analysed the gaze dwell time and the number of gaze movements to clarify what kind of influence and learning effect it has on the cognitive process.
\end{abstract}

Keywords-Highlighter pen; Learning strategy; Eye movement

\section{INTRODUCTION}

Currently, the mainstream of the Japanese educational policy is not to apply academic pressure on the students. Free education or cramming education systems are still highly recommended, but it is important to ensure that students have basic knowledge and skills first. Leveraging students' knowledge and skills, and fostering their power to express what they want, ultimately leads students to develop a "zest for living" [1]. In addition, the government course of study curriculum guidance will be effective in 2020. In order to foster students' abilities to solve questions, the introduction of active learning is considered to be effective to raise their creativities. In other words, it is a matter, of course, that students should have mastered the basic knowledge and academic ability prior to being introduced to active learning. Furthermore, there is a need for students to learn how to judge, express, and think by themselves. In order to achieve this goal, the learners should first master the basic knowledge and then, move on in mastering the learning strategies to associate the learning material with their knowledge.

In recent years, learning methods and learning systems focusing on the learner's cognitive processes are expected as one of the new learning strategies. The following are some learning strategies that have been examined. For example, the rehearsal strategy requires the process of repetition of memorised materials. And the refinement strategy connects the learning material and the already-known knowledge. Other examples are the iterative writing repetition strategy and the planning strategy to conduct learning based on the plan prepared in advance.

It was evident that learners, who had chosen appropriate learning strategies, deepened their learning content and acquired more knowledge compared to learners, who still was not sure of their learning strategies. However, regardless of any learning strategy, learners use various writing instruments, such as pencils, mechanical pencils, and ballpoint pens. Among these writing instruments, we focused on the highlighter pen. A highlighter pen is generally used for the purpose of prompting learners by colouring keywords or phrases of importance, thereby improving learning efficiency. It is thought that colouring and highlighting text with a highlighter pen will influence the learner's cognitive process in learning. Regarding the use of the highlighter pen for learning, the following research has been done so far. In the past, studies on the effect of highlighting by a highlighter pen on the colour and memory favourable to vision [2], the study of the influence of appropriate highlighting in sentences on keyword search, and the study on the learning effect with presence/absence of highlighting was conducted $[3,4,5]$. As a result of these studies, it was shown that the learning effect was observed as expected; however, the reason for this occurrence has not yet been confirmed. Also, it is not clarified what kinds of influence extended to the learner's attention, consciousness, memory and others by using a highlighter pen. And overall, it remains unclear of what kind of change was brought to the cognitive process.

In this study, we developed contents for learning based on our proposed learning strategies. Some researches focused on the eye movement for investigating the effect of presence/ absence of highlighting [6,7,8], but the training effect of highlighting was not mentioned. Therefore, we clarified the relationships and the changes between the improvement of the learner's performance, eye movement and gaze time before and after learning using that content.

\section{EXPERIMENTAL METHOD}

A. Gaze measurement experiment based on the presencelabsence of highlighting in problem sentence

1) Overview of Experiment

The learning strategy that is being proposed is highlighting keywords and numbers in the question text for deriving answers. As a consequence, respondents can organise information in the question and accurately recognise important information for answering the questions.

Experiments were conducted using two kinds of sentence problems that students learn in English: (1) questions involving third-person singular present tense, and (2) overall verb tense question sentences, such as past, present and future. The differences in gaze dwell time, the number of gaze movements 
to the keywords in the sentence problems were examined. In addition, the correct answer rate comparing the cases with highlighting and without highlighting was also examined.

\section{2) Experimental Conditions}

The experiment was conducted in a classroom of a cram school located in Higashiosaka city, Osaka, Japan. Subjects solved 30 questions in English, projected onto the whiteboard: 10 without highlighting, 10 with highlighting and 10 without highlighting. It was hypothesised that by highlighting keywords, students will become more aware of the keywords, and this effect would continue even after removing the highlighting. Since, there was a fear of students getting used to the questions, the questions of third-person singular present tense and the verb tense questions were asked alternately. Subjects orally answered as the next question was displayed simultaneously. Figure 1 shows the examples of the questions. For the questions regarding third-person singular present tense, the subjects were told to choose an answer from two options. Similarly, for questions revolving verb tense, the subjects were told to choose an answer from four options. In the case of highlighting being present, for the questions about third-person singular present tense, the students highlighted the subject of the sentence, and similarly for the questions about verb tenses, the verb tense related keywords were highlighted.

\begin{tabular}{|c|c|c|c|}
\hline \multicolumn{4}{|c|}{ Ms. Green (ア get $イ$ gets ) up at six. } \\
\hline Ken & $\begin{array}{l}\text { (1) helps } \\
\text { (2) helped } \\
\text { (3) will help } \\
\text { (4) help }\end{array}$ & his mother las & st Sunday. \\
\hline \multicolumn{4}{|c|}{ (a) Without Highlighting } \\
\hline \multicolumn{4}{|c|}{ We ( ア watch イ watches) TV every day. } \\
\hline She & $\begin{array}{l}\text { (1) practices } \\
\text { (2) will practice } \\
\text { (3) practiced } \\
\text { (4) practice }\end{array}$ & the piano e & everyday. \\
\hline
\end{tabular}

(b) With Highlighting

Fig. 1. Examples of English problems

\section{3) Subjects}

The subjects were 20 junior high school students: 11 boys and 9 girls. There were 3 second grade junior high school students and 17 third grade junior high school students. All subjects had normal eyesight with their naked eye or with use of a corrective lens, such as eyeglasses or contact lenses.

\section{4) Experimental Environment}

The problem was shown in the area of $80 \mathrm{~cm} \times 140 \mathrm{~cm}$ on the white board, and the height of the board was $95 \mathrm{~cm}$ from the floor as shown in Figure 2. The subjects were seated at a distance of $130 \mathrm{~cm}$ from the whiteboard, fixed to the head with the chin rest so that their heads would not move, and a gaze measuring device was attached as shown in Figure 3. Also, Figure 4 shows a picture of the chin rest that fixed the head. EMR-9 manufactured by Nac Image Technology Co., Ltd. was used as the visual axis measuring apparatus as shown in Figure 5 .

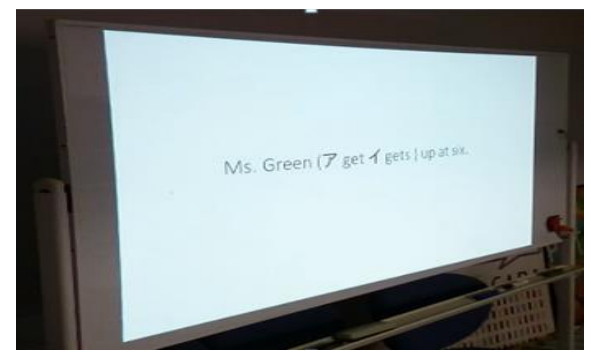

Fig. 2. Question Displayed on Whiteboard

The image of the visual field camera is displayed as shown in Figure 6; the eye mark of " $\square$ " indicates the gazing point of the right eye, and the eye mark of " + " indicates the gazing point of the left eye.

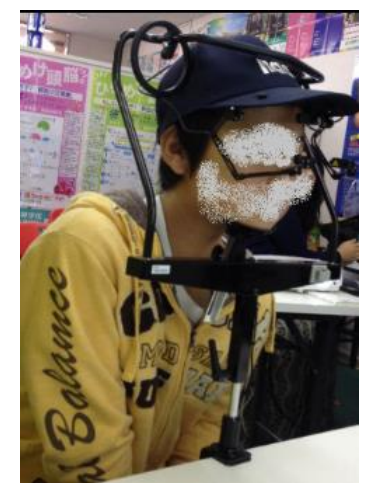

Fig. 3. Subject Wearing Gaze Measuring Device

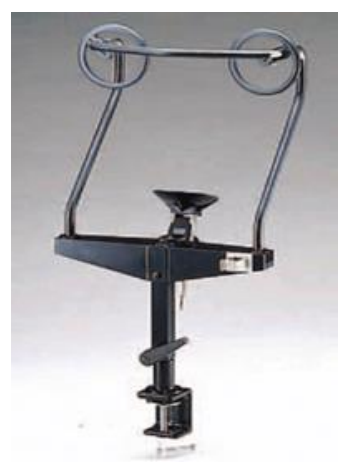

Fig. 4. Chin Rest

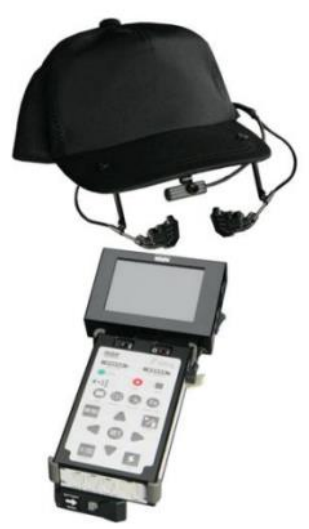

Fig. 5. EMR-9 


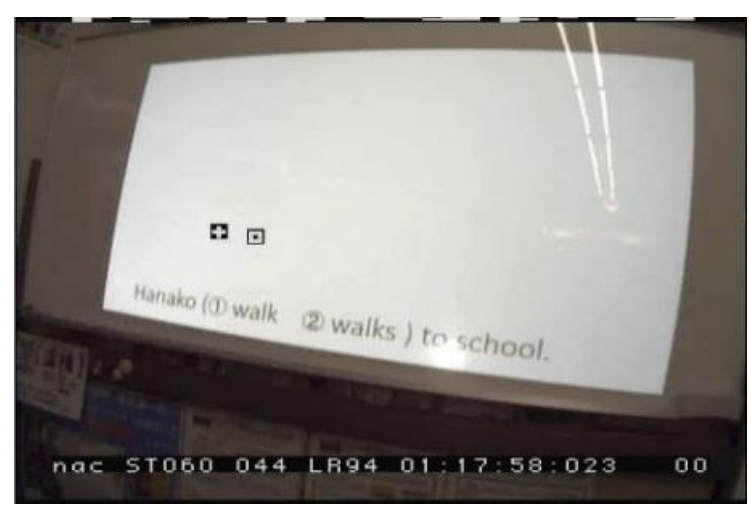

Fig. 6. Snapshot from Visual Field Camera

\section{5) Data Analysis Method}

For gaze point detection, a gaze counting software was used to superimpose the coordinate data of gaze point of each question, a snapshot of view camera, and a heat map was created. Next, the snapshots of the view camera were divided into the areas of words: subject, verb, time, and others. Then, the area of each word was selected by the operator and the number of gaze points in each area was counted as shown in Figure 7.

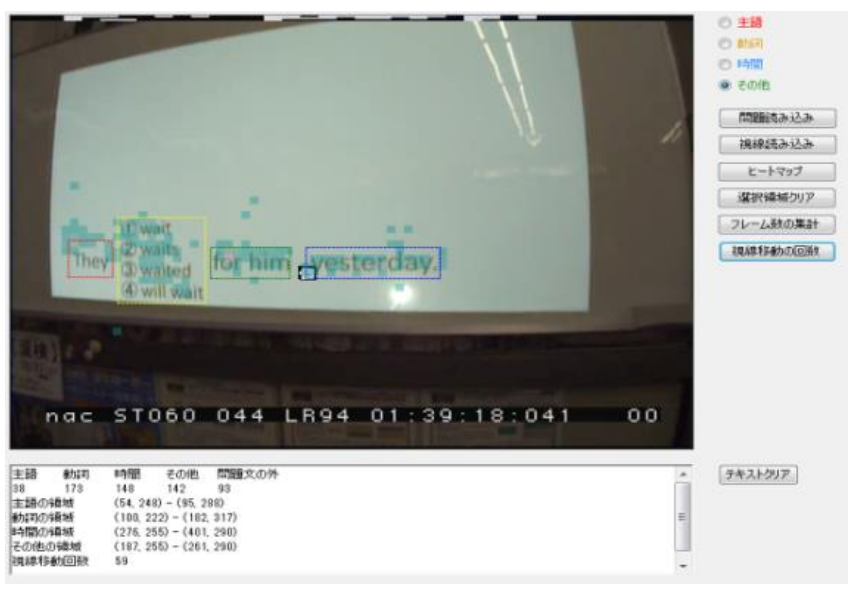

Fig. 7. Example of Data Analysis

\section{B. Experiment on training effect by our proposed highlighting method}

\section{1) Overview of Experiment}

The gaze measuring device used in the experiment of the previous section took time to calibrate and the head had to be fixed so some subjects felt distressed. A different device was used to carry out the experiment in this section. The device used in this experiment of this section was carried out by a device manufactured by Tobii Technology $A B$ which could perform the calibration relatively more smoothly and did not need to fix the head. Particularly, in the top-ranked and lowranked respondents, we examined whether the gaze movement and the number of gaze movements differed when solving the question. Also, by conducting training to highlight the appropriate keywords in the question text for a certain period of time, we verified the kind of learning effect present in the gaze data to low-ranked respondents.
Subjects were first asked 30 questions about verb tense (given 3 choices for answers). Then, the sentence in the question was divided into 4 categories: (1) subjects, (2) verbs, (3) words indicating time, and (4) other areas. Then they measured the gaze dwell time of each area and the number of gaze movements from the area to the other area. After that training was carried out with the same tense questions at apace of one training session every two days over the course of two weeks. And after these two weeks, the subject solved 30 questions repeatedly, and the gaze data at that time was measured. During this time, subjects answered the questions orally and then the following question was displayed. We also told the subjects to be conscious of answering as soon as possible and allowed the subjects to solve the sample question as practice, beforehand.

\section{2) Experimental Condition}

The subjects were seated at a distance of about $50 \mathrm{~cm}$ from the laptop computer screen which displayed English sentence problems. Then, they were asked to solve 30 English sentence problems displayed on the screen.

Subsequently, by excluding 3 subjects with high percentage of correct answers, 16 subjects from the original 19 subjects were further divided into two groups: (1) Group A, and (2) Group B. Group A consisted of people that do not use highlighting and Group B consisted of people that perform training using highlighting. Then, after training with similar English tense questions once every two days over the course of two weeks, subjects again solved 30 English questions of English verb tense sentence problems different from last time under similar conditions. The question used in the experiment is shown in Figure 8.

The hypothesis was that the training method proposed would improve gaze movements of the students and achievement of the test.

$$
\begin{aligned}
& \text { (1) helps } \\
& \text { Ken (2) helped his mother last Sunday. } \\
& \text { (3) will help } \\
& \text { (4) help }
\end{aligned}
$$

Fig. 8. Examples of Sentence Problems

\section{3) Subjects}

The number of subjects was 19: 10 boys and 9 girls in junior high school. All subjects had normal eyesight with their naked eye or with use of a corrective lens, such as eyeglasses or contact lenses.

\section{4) Experimental Environment}

Experiments were conducted at a cram school in HigashiOsaka city, Osaka, Japan. The subject was seated about $50 \mathrm{~cm}$ from the laptop computer screen and orally answered the displayed question. For questions switching operation, the monitor was prepared behind the subject and the operator performed the operation as shown in Figure 9. The device manufactured by Tobii Technology as a visual line measuring device was installed at the bottom of the display of the laptop computer as shown in Figure 10. 


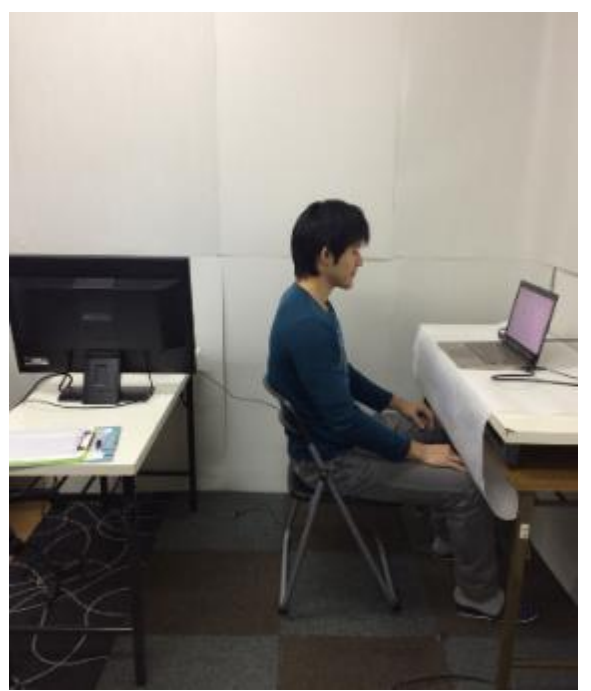

Fig. 9. Experiment Environment

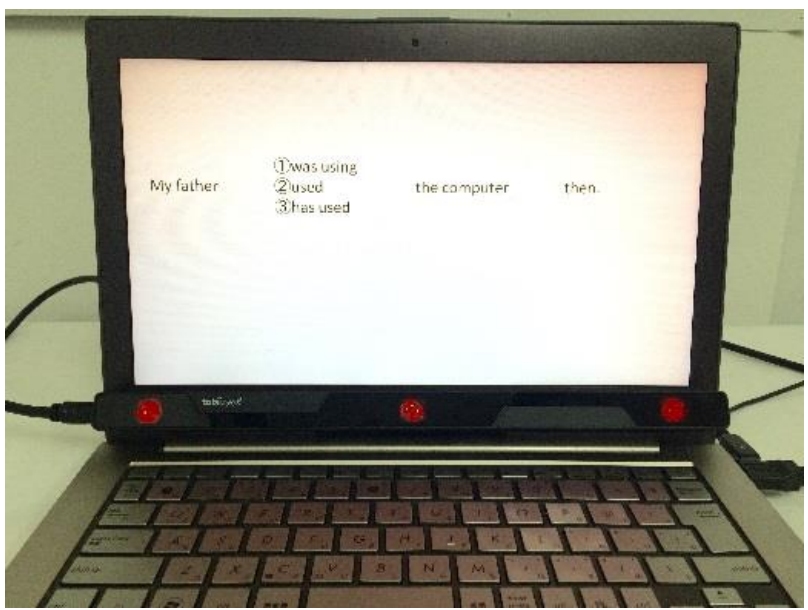

Fig. 10. Laptop Computer with Device of Tobii Technology

\section{5) Training Contents of Our Proposed Highlighting} Method

Subjects divided into Group A and Group B were asked to perform training by using 20 questions 8 times, that is, in other words, 160 questions in total. In Group A training, subjects selected the answer to the displayed question and answered on a separate answer sheet. In Group B training, subjects drew a line with their finger with a highlighting function for the part considered as the keyword of the question text displayed on the tablet while subjects subsequently answered the question. The training content is shown in Figure 11. When moving onto the next screen, a new question is displayed to the subject, and the subject would think and answer the place to highlight on the tablet. Figure 12 shows the training situation for Group B.
1. Question is displayed on the screen.

I (1)write (2)will write (3)have written) a letter to my grandmother tomorrow.

2. The student highlights keywords, and answer the question.

\section{I (1) write (2)will write (3)have written) a letter to} my grandmother tomorrow.

\section{Correct keywords are highlighted, and the answer is displayed.}

\section{I (1)write (2)will write (3)have written) a letter to my grandmother tomorrow.}

Fig. 11. The Sequence of the Training for Group B

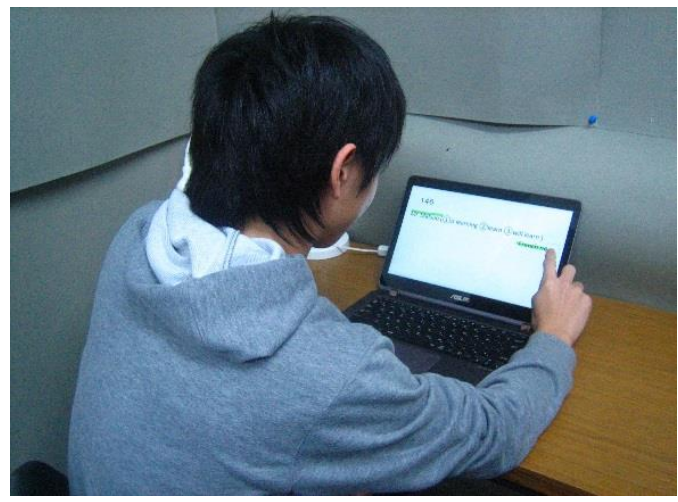

Fig. 12. Training Situation for Group B

6) Data Analysis Method

Regarding the analysis, the coordinate areas of the words constituting each problem were measured beforehand, and compared with the coordinate data of the attention points obtained in the experiment, the gaze points were calculated. It has been reported that people can recognise an English word in approximately 50 milliseconds to 60 milliseconds. Therefore, when a gaze point exists on a specific word for 60 milliseconds or more, it is set as a stationary point. Based on the results, the gaze dwelling time and the number of gaze movements were calculated. 


\section{EXPERIMENTAL RESULT}

A. Gaze measurement experiment based on the presencelabsence of highlighting in problem sentence

The experimental results for the experiment described in Section 2.1 are shown below. Since the marking places are different between the third-person singular present tense and the verb tense questions, the analysis results were divided for each. First, with respect to line-of-sight data at the time required for solving questions, the average number of gaze movements is shown in Figure 13 and Figure 14.

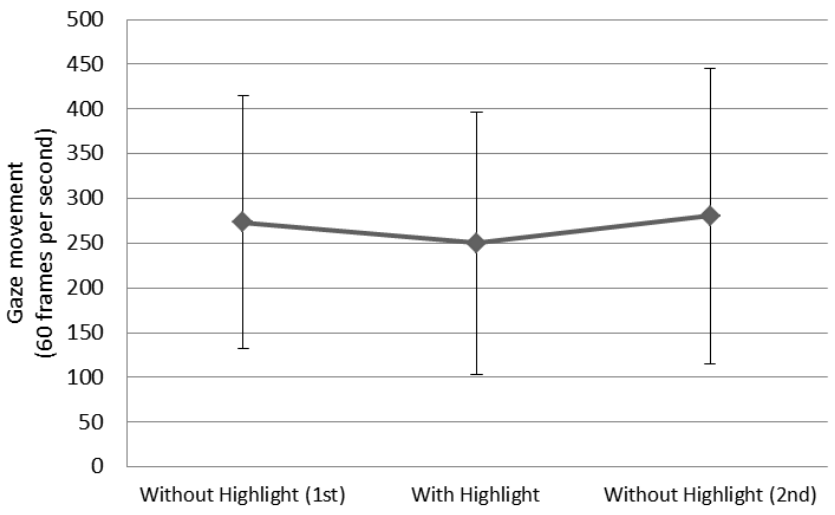

Fig. 13. Number of Gaze Movements in case of Third-person Singular Present Tense Sentence Problems

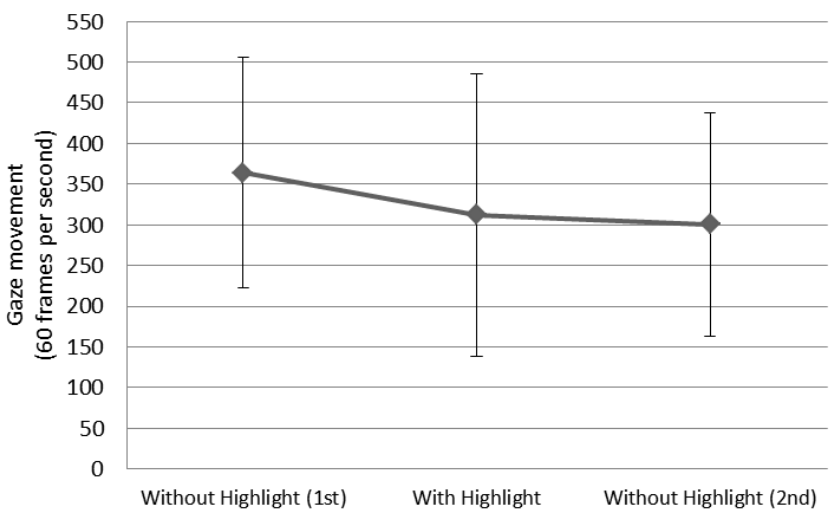

Fig. 14. Number of Gaze Movements in case of Verb Tense Type Questions

It is suggested that movement of the line of sight was affected by highlighting, because the number of gaze movements tends to decrease with highlighting condition in both cases for questions revolving the third-person singular present tense and verb tense. However, when the t-test was performed, no significant differences were found in either cases ( $p>0.05$ ). Also, the effect of highlighting was not observed in third-person singular present tense type questions, because the number of gaze movement increased in withouthighlight (2nd) condition. Next, the number of frames stayed in each area of subject, verb, time and others, while questions solving was counted in each case. The results are shown in Figure 15 and Figure 16, respectively. The number of gaze frames in the verb area where options were presented, was the largest in both cases.
Also, with highlighting, the subject area was the second most frequently gazed while solving third-person singular present tense problems with highlighting. In the verb tense type questions, the time area is the second most frequently gazed when highlighting was present. In both cases of the thirdperson singular present tense and verb tense type questions, the other area that was not important for solving the questions was gazed more in the "without-highlighting" condition rather than in the "with-highlighting" condition.

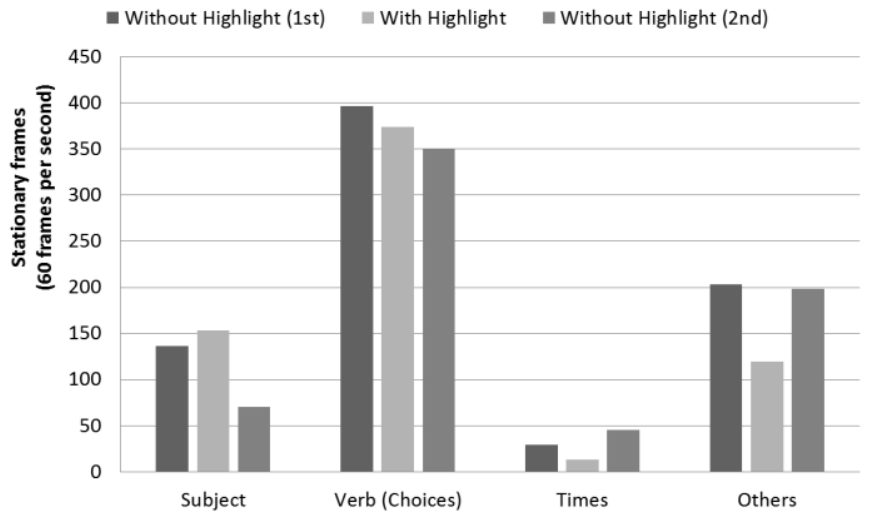

Fig. 15. Number of Stationary Frames Incase of Third-person Singular Present Tense Type Questions

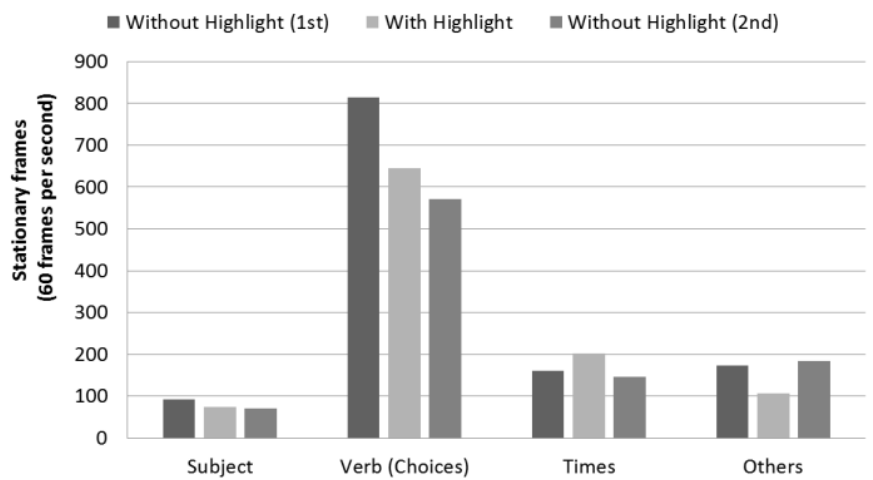

Fig. 16. Number of Stationary Frames Incase of Verb Tense Problems

From these results, it is considered that highlighting had an effect on the cognitive process, while the students were solving questions. However, there was no significant difference in either cases $(\mathrm{p}>0.05)$.

We anticipated that upper grade students already had acquired efficient answering strategies. On the other hand, lower grade students tended to gaze at places unrelated to solving the questions. Therefore, the number of gaze movements in case of the third-person singular present tense type questions and the verb tense type questions are shown in Figure 17 and Figure 18, respectively. The total average is shown in Figure 19.

In both cases of question types and highlighting conditions, the number of gaze movements was smaller in the students in the upper grade compared to the students in the lower grade. For the upper grade, the number of gaze movements was 
smaller with highlighting than without highlighting. On the other hand, for students in the lower grade, the number of gaze movements was more or less about the same or even larger with highlighting than without highlighting.

For the students in the lower grade, it seemed to be insufficient to highlight the important part of the question text in order to achieve a higher score. Therefore, we develop the online contents for training the students and investigated the effect of the training.

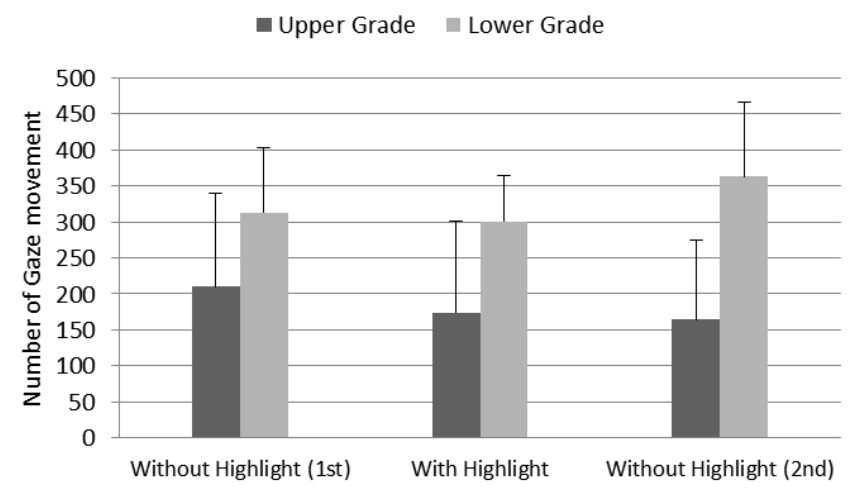

Fig. 17. Number of Gaze Movements in case of Third-person Singular Present Tense Type Questions

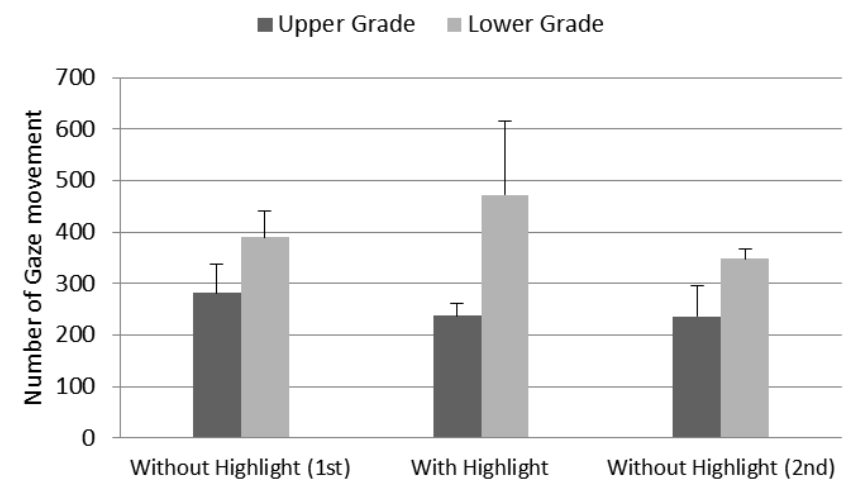

Fig. 18. Number of Gaze Movements in case of Verb Tense Type Questions

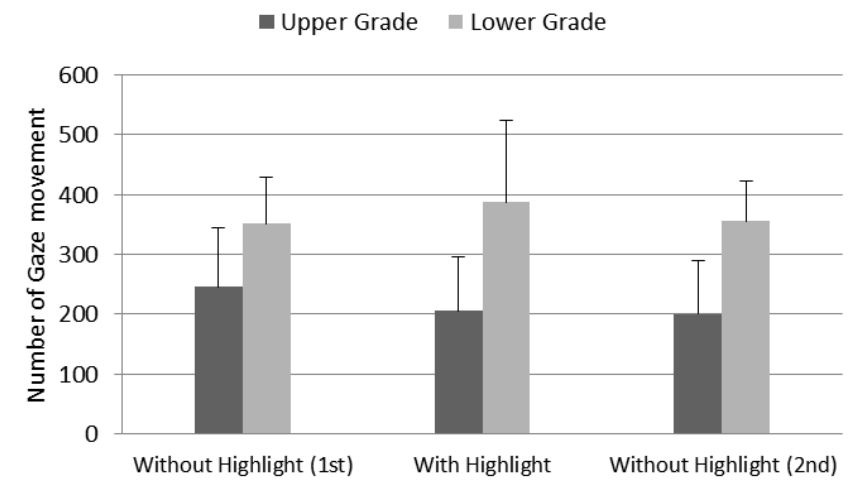

Fig. 19. Total Average Number of Gaze Movements

\section{B. Experiment on training effect by our proposed highlighting method}

The results of the experiment described in Section 2.2 are shown below. Figure 20 shows the comparison of the correct answer rate before training, expressed as "pre", and after training, (expressed as "post"). The percentage of correct answers from "pre" to "post" improved by $10 \%$ in Group A and by $10.8 \%$ in Group B. In addition, although the full score was not in "pre" portion, there were six full-score people in the "post" portion in which two people were from Group A and four people were from Group B.

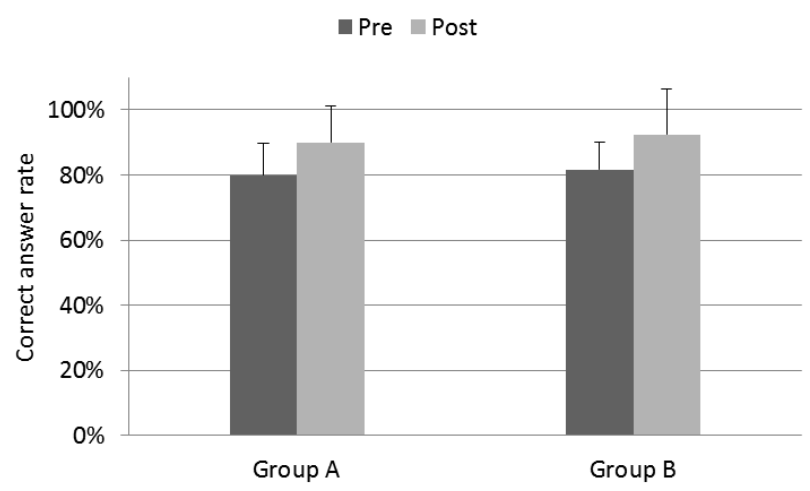

Fig. 20. Correct Answer Rate in Pre- and Post- conditions

Figures 21 and 22 shows the number of gaze movements and the number of stationary frames of verb area where the options were presented. In both Groups A and B, the number of gaze movements and the number of stationary frames decreased as the experiment went on from "pre" to "post" conditions. In Group $\mathrm{A}$, the number of gaze movements decreased by 44.3 , while the number of stationary frames also decreased by 142.5. In Group B, the number of gaze movements and the number of stationary frames also decreased, while they decreased by 57.6 and 172.5 , respectively. In both cases, Group B gained a trend that the rate of decrease in the number of gaze movements and the number of stationary frames was higher.

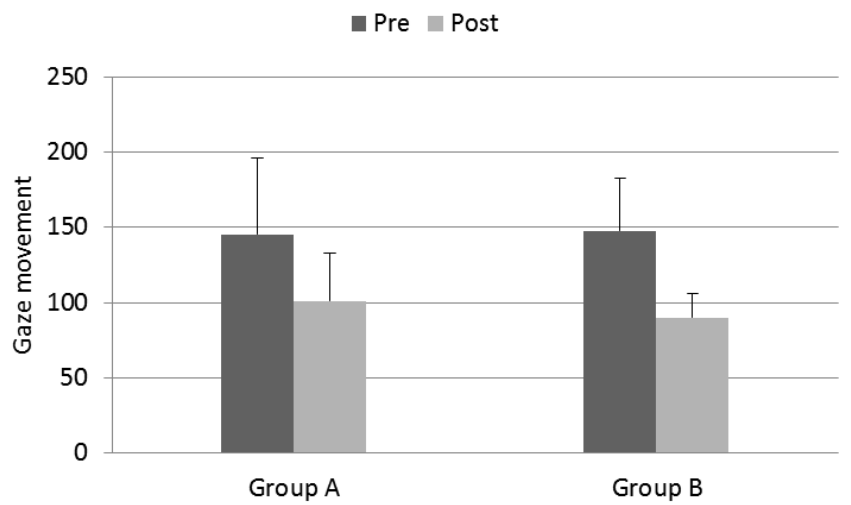

Fig. 21. Number of Gaze Movements in Pre- and Post-conditions 


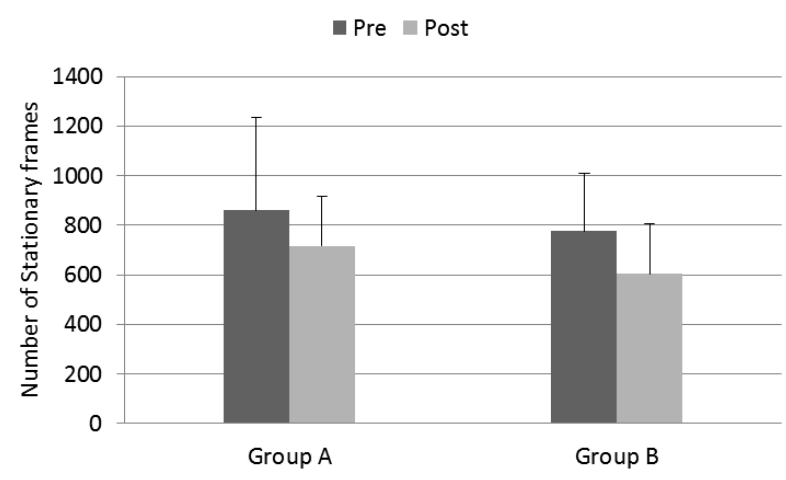

Fig. 22. Number of Stationary Frames in Pre- and Post-conditions

Next, we examined how these numbers decreased in terms of the achievement, in other words, correct answer rate of "pre" test. We defined the degree of decreasing of numbers was defined as a "pre" number divided by "post" number. Figure 23 shows the scatter plot of the correct answer rate versus the degree of decreasing of number of gaze movements. And Figure 24 shows the scatter plot of correct answer rate versus the number of degree of decreasing of stationary frames on verb area.

Group A showed no correlation between "pre" test achievement and the degree of decreasing number of gaze movements. On the other hand, Group B showed moderate correlation $(\mathrm{r}=0.45)$. Group A showed moderate correlation between "pre" test achievement and the degree of decreasing number of stationary frames $(\mathrm{r}=0.52)$, while Group B showed no correlation.
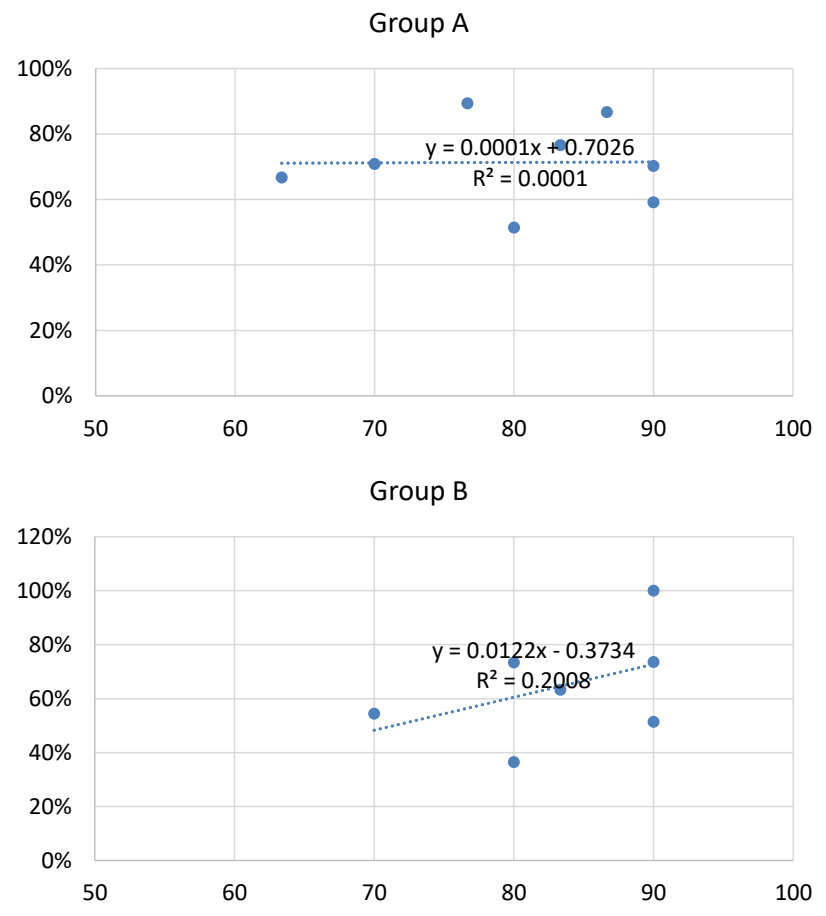

Fig. 23. Correct Answer Rate vs. Degree of Decreasing of Number of Gaze Movements
The training method used in Group B that we propose showed the effectiveness for the students in the lower grade in terms of decreasing number in gaze movements. This means that the students in the lower grade were able to improve their searching skills for finding important information in a sentence problem. On the other hand, the conventional learning method used in Group A shows effectiveness for students in the upper grade in terms of decreasing number of stationary frames. This means that students in the upper grade were able to improve their speed in choosing the answer from options.

\section{CONCLUSION}

Although there are various strategies when the learner learns, the purpose of this study is to focus on the cognitive process of learning by the learner and to train their learning by using the highlighter pen. This experiment was conducted so that the change in the correct answer rate of a question can be examined. We conducted this experiment so that we can examine whether there is a change in the correct answer rate of a problem or a change in the movement of the line of sight between the two groups: (1) students in the upper grade, and (2) students in the lower grade.

In experiments using highlighting, it was found that there was a difference between the number of eye movements and the time that took students to look at keywords on questions with and without highlighting. It is thought that because the highlighting was given to the appropriate place, it enabled students to answer the questions correctly with the shortest path.
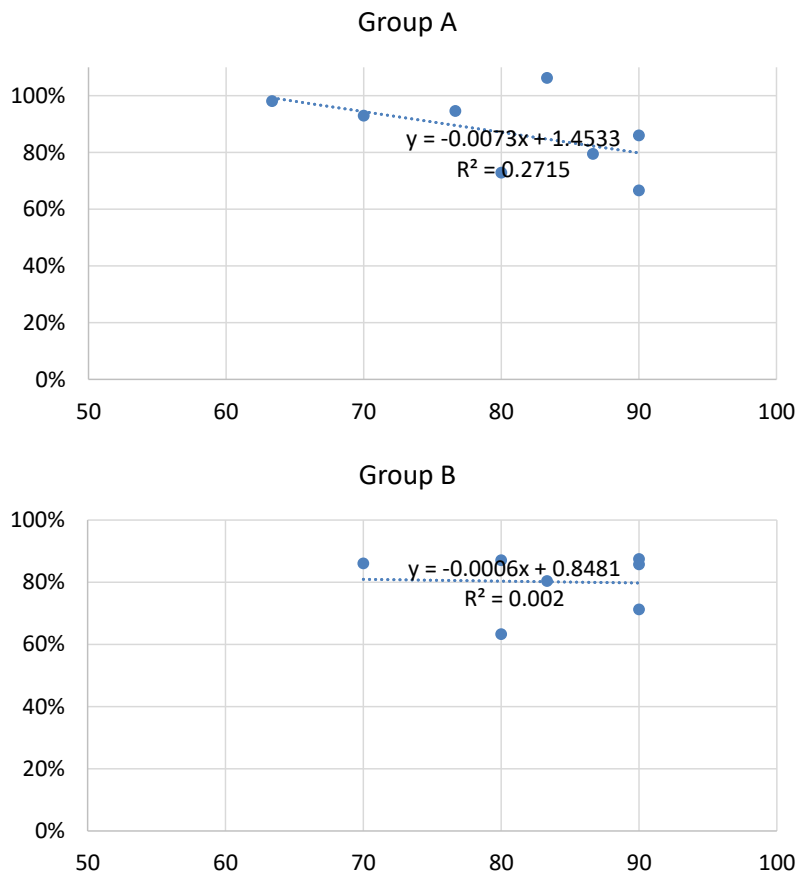

Fig. 24. Correct Answer Rate vs. Degree of Decreasing of Number of Gaze Movements

In the verification experiment of the training effect, training showed a tendency that the number of gaze movements and the gaze dwell time to the verb decreased, and the correct answer 
rate increased. In addition, the students in the lower grade tended to have a shorter number of gaze movements by our proposed training method using the highlighter pen. Therefore, it was suggested that the students in the lower grade will improve their achievement level by continuing the training.

We continue to investigate the effectiveness of our proposed training method by observing physiological index such as electroencephalogram, electrocardiogram, heartbeat, respiratory rate, and skin potential.

\section{ACKNOWLEDGEMENTS}

We are deeply grateful to Mr. Kazumasa Shibata, and Ms. Yuki Inazuka. Without their dedicated support, this paper would not have materialised.

\section{REFERENCES}

[1] HP of Ministry of Education, Culture, Sports, Science and Technology: http://www.mext.go.jp/b_menu/shingi/old_chukyo/old_chukyo_index/to ushin/attach/1309612.htm (in Japanese)
[2] R. Suzuki, M. Kimura, Y. Horie, and H. Ohuchi, "The effect of marking by the fluorescent color," Proceedings of Annual convention of Japan Ergonomics Society, Vol.38, pp.500-501, 2002.

[3] H. Nishimura and N. Kuwahara, "A Study on Learning Effects of Marking with Highlighter Pen," Springer Lecture Note on Computer Scinece, volume 9184, pp 357-367, 2015.

[4] Carole L. Yue, Benjamin C. Storm, Nate Kornell, and Elizabeth Ligon Bjork, "Highlighting and Its Relation to Distributed Study and Students' Metacognitive Beliefs", Educational Psychology Review, March 2015, Volume 27, Issue 1, pp 69-78

[5] Fowler, Robert L.; Barker, Anne S, "Effectiveness of highlighting for retention of text material", Journal of Applied Psychology, Volume 59, Issue 3, Jun 1974, pp358-364.

[6] H. Nishimura, K. Shibata, Y. Inazuka and N. Kuwahara, "A Study of Eye Movement Analysis for Investigating Learning Efficiency by Using a Highlighter Pen," Springer Lecture Note on Computer Scinece, volume 9745, pp 576-585, 2016.

[7] Ed H. Chi, Michelle Gumbrecht, and Lichan Hong, "Visual Foraging of Highlighted Text: An Eye-Tracking Study", Springer Lecture Note on Computer Scinece, volume 4552, pp 576-585, 2007.

[8] Hector R. Poncea, and Richard E. Mayer, "An eye movement analysis of highlighting and graphic organizer study aids for learning from expository text", Computers in Human Behavior, Volume 41, December 2014, pp.21-32 